\title{
The Decay of a Spark Channel which has ceased to carry Current
}

\author{
By D. C. CREAGH, N. H. FLETCHER AND J. M. SOMERVILLE \\ Department of Physics, University of New England, Armidale, N.S.W., Australia \\ MS. received 25th July 1962
}

\begin{abstract}
The method used by Somerville and Williams to study the development of a spark column by passing through it a delayed current pulse has been extended so that the whole evolution and decay of the channel may be examined. The column produced by a $32 \mathrm{~A}$ spark in air with duration less than $100 \mathrm{nsec}$ is found to expand initially in close association with a cylindrical shock wave; a period of slower expansion follows in which, presumably, conditions within the column approach pressure equilibrium. Finally the column cools and contracts by a process of radial thermal conduction. This third stage has been treated theoretically and an expression for the radius of the column as a function of time has been derived which gives satisfactory agreement with experiment.
\end{abstract}

\section{§ 1. INTRODUCTION}

COMERVILLE and Williams (1959) have described a method of studying the initial Nexpansion of the conducting region of a spark channel. The channel is initiated by the passage across a gap of a rectangular current pulse, called the 'initiating' pulse. After the passage of this pulse the channel expands freely until, at a controlled time later, a second 'probing' pulse is passed through the gap. If a specially treated anode is used, the probing pulse leaves on it a number of small spots distributed over a circular area the radius of which is taken to be the radius of the conducting channel at the time of the passage of the probing pulse. Using initiating and probing pulses with currents of about $30 \mathrm{~A}$ and duration of about $30 \mathrm{nsec}$, and with delay times between the pulses of up to $1 \mu \mathrm{sec}$, the initial period of expansion of a spark channel in air was investigated at pressures between atmospheric and about $200 \mathrm{mmHg}$.

The same method may be used to study the decay of the conducting channel, provided that the delay between the initiating and probing pulses can be increased to some tens of microseconds. This paper describes an investigation of channel decay by this method.

\section{§2. APPARATUS}

Somerville and Williams (1959) used equipment described by Somerville (1959) in which the initiating pulse was reflected from the shorted end of a coaxial line and returned through the channel to serve again as the probing pulse. This arrangement was suited to short delay times of the order of a microsecond or less, but it is not convenient when longer delays are required. Consequently, for the present study, different equipment was built for generating the initiating and probing pulses. Figure 1 is a schematic diagram of this equipment.

$\mathrm{G}_{1}$ is the gap across which the spark channel to be studied is established. It is enclosed in a spark chamber which can be evacuated or pressurized, and the length of 
the gap may be varied by means of a micrometer. The anode $\mathrm{A}$ is a flat circular disk. The cathode $\mathrm{C}$ is a hemispherically ended tod $2 \mathrm{~mm}$ in diameter which may be placed in several different positions radially along the arm $B$. By rotating $B$ about the spindle $D$ and varying the position of $\mathrm{C}$ the marks left by many sparks may be recorded on a single anode disk.

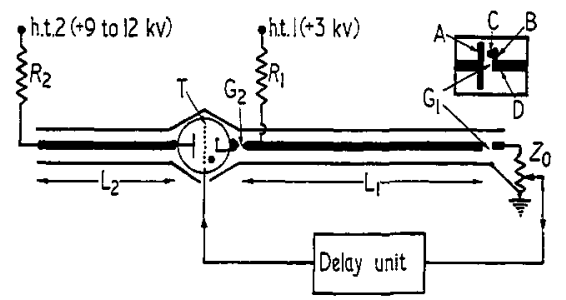

Figure 1. Schematic diagram of the double-pulse apparatus. The initiating pulse is formed by the discharge of line $L_{1}$ through the test gap $G_{1}$. The probing pulse formed by the discharge of line $\mathrm{L}_{2}$ also passes through $\mathrm{G}_{1}$ after first passing through the thyratron $T$ and the subsidiary gap $G_{2}$. The inset shows details of the electrode arrangement in the test gap $\mathrm{G}_{1}$.

The initiating current pulse is formed by the discharge of the coaxial line $L_{1}$ through $G$ into its characteristic impedance $Z_{0}(47 \Omega)$. The length of $L_{1}$ is variable and initiating pulse durations of 40,47 and $60 \mathrm{nsec}$ may be obtained. $\mathrm{G}_{1}$ is broken down by first applying the full high-tension voltage h.t.1, and then moving the electrodes together until the sparking distance is reached. At the line voltage used $(3 \mathrm{kv})$ this distance is approximately $0.8 \mathrm{~mm}$. At the end of the initiating pulse the centre conductor of the line $L_{1}$ is at zero potential and its recharging time through $R_{1}(0.5 \mathrm{sec})$ is so long that it remains substantially at zero potential during the delay between the initiating and probing pulses.

Part of the voltage pulse appearing across $Z_{0}$ is used to trigger the delay unit, the output of which is applied to the grid of the BT 101 hydrogen thyratron $T$. When T fires, the line $L_{2}$ discharges through the subsidiary gap $G_{2}$ into the line $L_{1}$ and thence through the conducting channel across $G_{1}$, and this discharge forms the probing pulse. The discharge of $L_{2}$ gives a reactangular probing pulse of $40 \mathrm{nsec}$ duration, but other pulse-forming networks or impedances may be connected to the open end of $L_{2}$ to give probing pulses of different durations and shapes.

The delay unit consists of a flip-flop stage followed by a BT 79 thyratron which provides the grid pulse required to fire the thyratron $T$. The gap $G_{2}$, which serves to eliminate any leakage currents through $\mathrm{T}$ from its high-tension supply h.t.2 to the central conductor of $L_{1}$, is irradiated with ultra-violet light to reduce the statistical lag of breakdown of the gap to a negligible amount. With this arrangement overall delays between initiating and probing pulses of from $1.5 \mu \mathrm{sec}$ to $20 \mathrm{msec}$ may be obtained. By by-passing the flip-flop stage and firing the BT 79 thyratron through a length of coaxial cable from part of the pulse across $Z_{0}$, it is possible to obtain delays as low as $350 \mathrm{nsec}$. In order to reduce the delays in firing the thyratrons, each is supplied with a grid voltage pulse in excess of the normal rated value.

\section{§3. EXPERIMENTAL PROCEDURES AND RESULTS}

The palladium anode was polished by a method described by Stanton (1958) and then coated with 21 layers of barium stearate by the Langmuir-Blodgett process (Langmuir 1939). In all the work described here, the gas between the electrodes was air at 
atmospheric pressure. The anode marks were observed with a phase-contrast microscope fitted with a graticule containing a number of concentric circular rings; the radius of the smallest circle containing all the spots made by the probing pulse was measured and the number of spots in each annulus between graticule rings was counted. Means of these quantities, measured for at least 20 marks made by sparks under the same conditions, were taken. The initiating pulse current was kept at $32 \mathrm{~A}$. Experiment showed that the radius of the anode mark left by the probing pulse was quite insensitive to the probing pulse duration and amplitude, and a duration of about $100 \mathrm{nsec}$ and an amplitude of about $120 \mathrm{~A}$ were used throughout.

Figure 2 shows the conducting channel radius $R$ at time $t$, deduced from the anode marks obtained with the apparatus described above, with initiating pulse durations of 40,47 and $60 \mathrm{nsec}$. These measurements were perforce confined to values of $t$ exceeding

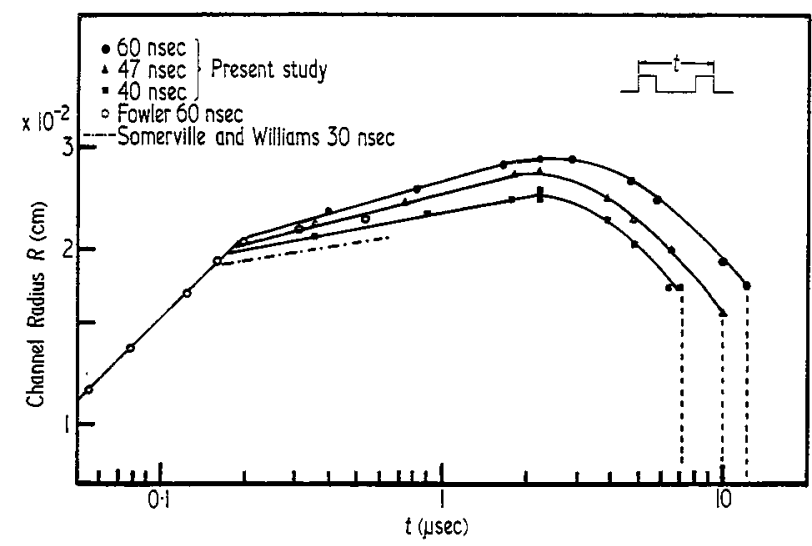

Figure 2. Summary of measurements showing the complete history of the spark channel. The initial expansion as $t^{1 / 2}$, the intermediate region, and the final thermal decay are clearly seen. Vertical broken lines indicate times of apparent extinction of the channel. Initiating pulse lengths are given in the key.

about 350 nsec. For comparison, two sets of measurements of channel radii for smaller values of $t$ are shown: one set of the original measurements of Somerville and Williams (1959) corresponding to a $30 \mathrm{nsec}$ initiating pulse; and a similar set, made in the same way for us by Mr. D. K. Fowler, corresponding to a $60 \mathrm{nsec}$ initiating pulse. It is seen that there is a satisfactory join between the measurements of Fowler and of the present study.

In figure 2 the whole life of a channel initiated by a 60 nsec pulse may be followed. Up to about 100 nsec, the channel expands with $R \propto t^{1 / 2}$, which is consistent with its coincidence with a cylindrical shock wave generated by an instantaneous energy release, as discussed by Somerville and Williams (1959). After that time, the channel separates from the shock and expands much more slowly until about $3 \mu \mathrm{sec}$, when it begins to contract. The contraction proceeds until a point is reached where, so far as the method of study here is concerned, it 'disappears'. Observationally, what happens is that, after a certain time, the application of the probing pulse causes a fresh breakdown to occur, no longer related to the original channel. These points of extinction of the channel are indicated in figure 2 by the vertical broken lines at the ends of the curves.

The anode marks also yield interesting information about the distribution of conductivity across the channel during its life. At times up to about $5 \mu \mathrm{sec}$ after the initiating pulse the annular structures, first observed by Somerville and Grainger (1956), 
which suggest that most of the conductivity is concentrated near the periphery of the channel, are apparent. For longer delays this is no longer so and the region of maximum conductivity appears to move back towards the centre of the channel.

\section{§4. THEORETICAL DISCUSSION}

The history of the arc channel, as indicated above, may be conveniently considered in three periods. Initially the channel expands, following the shock wave, in a way which has been treated theoretically by Drabkina (1951). After the channel has fallen well behind the shock front there follows a period of slower expansion in which, presumably, adial pressure gradients are largely eliminated. The final period is one in which the channel, now approximately in pressure equilibrium, cools slowly towards room temperature. Since it is with this third stage that this study is primarily concerned it is conrenient to replot the data of figure 2 on a linear time scale. This is done in figure 3.

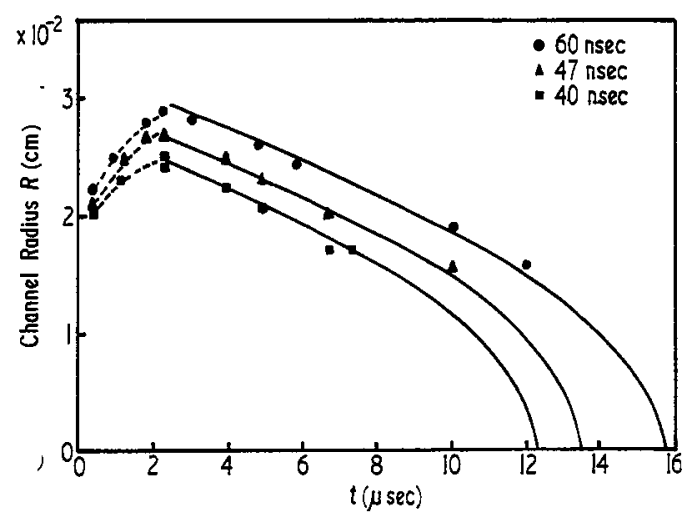

Figure 3. Some of the data of figure 2 replotted on a linear time scale. Full lines are theoretical curves calculated from equation (10) with the parameter $\tau$ equal to $14.5 \mu \mathrm{sec}$ and with central temperatures $T_{1}$ equal to $10000^{\circ} \mathrm{K}, 8500{ }^{\circ} \mathrm{K}$ and $7800{ }^{\circ} \mathrm{K}$ for the $60 \mathrm{nsec}, 47 \mathrm{nsec}$ and $40 \mathrm{nsec}$ curves respectively. Initiating pulse lengths are given in the key.

To examine the cooling of the channel theoretically, it is convenient, initially, to neglect end effects and to consider the thermal behaviour of an infinite cylinder of plasma of radius $R$, the pressure throughout being constant. In the type of atmosphericpressure arc which we are considering, the heat loss by radiation is relatively small and can reasonably be neglected in comparison with heat loss by conduction. Let the enthalpy of the plasma per unit mass be $H(T)$, the density $\rho(T)$ and the thermal conductivity $\kappa(T)$. Equating the rate of increase of the total heat content of the cylinder to the rate at which it gains heat, both by conduction and by influx of material, we obtain

$$
\frac{d}{d t} \int_{0}^{R} 2 \pi r \rho H d r=\left.2 \pi R \kappa_{R} \frac{\partial T}{\partial r}\right|_{r=R}+H_{R} \frac{d}{d T} \int_{0}^{R} 2 \pi r \rho d r .
$$

After some manipulation this can be expressed in the form

$$
\left.R \kappa_{R} \frac{\partial T}{\partial r}\right|_{r=R}=\frac{d}{d t} \int_{0}^{R}\left(H-H_{R}\right) \rho r d r+\frac{d H_{R}}{d t} \int_{0}^{R} \rho r d r .
$$

If now we fix attention on a particular value $T_{0}$ of $T$ and follow the way in which 
the value of $R$ appropriate to this temperature changes with time, we have

so that (1) becomes

$$
\frac{d H_{R}}{d t}=0
$$

$$
\left.R_{\kappa_{R}} \frac{\partial T}{\partial r}\right|_{r=R}=\frac{d}{d t} \int_{0}^{R}\left(H-H_{R}\right) \rho r d r .
$$

An approximate solution to this equation, giving $R$ as a function of $t$, can be obtained by expanding $H-H_{R}$ and $\rho$ in a Taylor series about $r=R$ and performing the integration. The resulting expression involves derivatives of both $H$ and $\rho$ with respect to radius (which can be converted to derivatives of $H$ and $\rho$ with respect to temperature), together with various derivatives of temperature with respect to radius at $r=R$. The solution becomes explicit if the radial temperature profile can be expressed in terms of one or more parameters which depend upon time only.

Measured radial temperature profiles for unconfined arcs show a general bell-like shape. For our purposes this profile may be conveniently approximated by a parabola or by a straight line, the latter approximation being more appropriate towards the edge of the arc column.

Referring to figure 4 , we see that, for a linear profile with central temperature

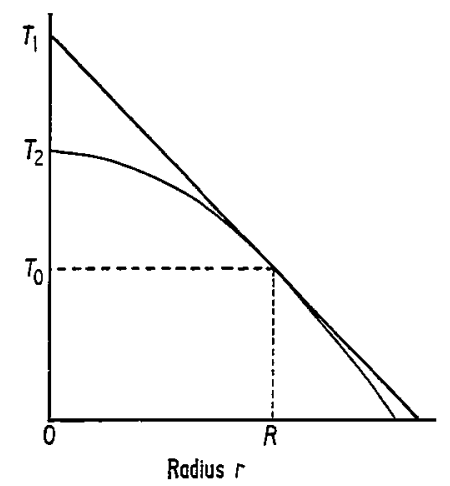

Figure 4. Linear and parabolic radial temperature profiles.

$T_{1}(t)$, the only non-zero derivative is

$$
\left.\frac{\partial T}{\partial r}\right|_{r=R}=-\frac{T_{1}-T_{0}}{R}
$$

while for a parabolic profile with central temperature $T_{2}(t)$, where

$$
T_{2}=\frac{1}{2}\left(T_{0}+T_{1}\right)
$$

the only non-vanishing derivatives are

$$
\left.\frac{\partial T}{\partial r}\right|_{r=R}=-\frac{T_{1}-T_{0}}{R},\left.\frac{\partial^{2} T}{\partial r^{2}}\right|_{r=R}=-\frac{T_{1}-T_{0}}{R^{2}} .
$$

Both these profiles thus involve only the single parameter $T_{1}(t)$.

Substituting (3) or (5) in the expanded form of the integral in (2), we obtain 


$$
\kappa\left[T_{1}(t)-T_{0}\right]=-\frac{d}{d t}\left\{R^{2} \sum_{n=1}^{\infty} a_{n}\left[T_{1}(t)-T_{0}\right]^{n}\right\}
$$

which can be written

$$
R^{2}=\frac{C-\kappa \int\left[T_{1}(t)-T_{0}\right] d t}{\sum_{n=1}^{\infty} a_{n}\left[T_{1}(t)-T_{0}\right]^{n}}
$$

where the constant $C$ is determined by the repuirement that the numerator vanish when $T_{1}(t)=T_{0}$, and the coefficients $a_{n}$ are given explicitly in terms of derivatives of $H$ and $\rho$ with respect to temperature. The coefficients $a_{n}$ differ, though not greatly, between the two assumed profiles. Their approximate values are listed, for $n \leqslant 6$, in the table. For a reason which will become apparent later, only first and second derivatives of $H$ have been retained. The terms of the series oscillate in sign and the series converges fairly rapidly, so that six terms are usually adequate.

\section{Approximate Values of Coefficients $a_{n}$}

\begin{tabular}{lll}
$n$ & \multicolumn{1}{c}{ Linear profile } & \multicolumn{1}{c}{ Parabolic profile } \\
1 & $\frac{1}{6} H^{(1)} \rho$ & $\frac{1}{8} H^{(1) \rho}$ \\
2 & $\frac{1}{12}\left(H^{(1)} \rho^{(1)}+\frac{1}{2} H^{(2)} \rho\right)$ & $\frac{1}{24}\left(H^{(1)} \rho^{(1)}+\frac{1}{2} H^{(2)} \rho\right)$ \\
3 & $\frac{1}{40}\left(H^{(1)} \rho^{(2)}+H^{(2)} \rho^{(1)}\right)$ & $\frac{1}{128}\left(H^{(1)} \rho^{(2)}+H^{(2)} \rho^{(1)}\right)$ \\
4 & $\frac{1}{60}\left(\frac{1}{3} H^{(1)} \rho^{(3)}+\frac{1}{2} H^{(2)} \rho^{(2)}\right)$ & $10^{-3}\left(2 \cdot 08 H^{(1)} \rho^{(3)}+3 \cdot 1 H^{(2)} \rho^{(2)}\right)$ \\
5 & $\frac{1}{504}\left(H^{(1)} \rho^{(4)}+H^{(2)} \rho^{(3)}\right)$ & $10^{-4}\left(-7 \cdot 8 H^{(1)} \rho^{(4)}-16 \cdot 7 H^{(2)} \rho^{(3)}\right)$ \\
6 & $\frac{1}{1344}\left(\frac{1}{5} H^{(1)} \rho^{(5)}+\frac{1}{2} H^{(2)} \rho^{(4)}\right)$ & $10^{-4}\left(1 \cdot 49 H^{(1)} \rho^{(5)}+3 \cdot 72 H^{(2)} \rho^{(4)}\right)$
\end{tabular}

Superscripts in parentheses indicate differentiation with respect to temperature at $T=T_{0}$.

In equations (3)-(5), $T_{1}(t)$ is an undetermined function of time. Its form can, however, be determined from equation (6). Suppose we expand $T_{1}(t)$ as

$$
T_{1}(t)=T_{1}+\left(\frac{d T_{1}}{d t}\right) t+\ldots
$$

and keep for the moment just these two terms, writing for convenience

$$
T_{1}(t)=T_{1}\left(1-\frac{t}{\tau}\right)
$$

The numerator of (6) can then be evaluated, along with the constant $C$, to give

$$
\frac{\kappa \tau}{2 T_{1}}\left[T_{1}\left(1-\frac{t}{\tau}\right)-T_{0}\right]^{2} .
$$

A factor $T_{1}(t)-T_{0}$ can be removed from the denominator leaving a quotient $S(t)$ which, ior small $T_{1}-T_{0}$, is approximately equal to $a_{1}$. Setting $t=0$ then, we find for the initial 
decay parameter $\tau$

$$
\tau=\frac{2 S R(0)^{2} T_{1}}{\kappa\left(T_{1}-T_{0}\right)}
$$

where $R(0)$ is the value of $R$ at $t=0$. Thus the initial value of $d T_{1} / d t$ is determined once the plasma column is described physically by giving $T_{1}$ and $R(0)$.

A further examination of equation (6) shows that $\left|d T_{1} / d t\right|$ decreases as $t$ increases, and it is clear physically that it must tend asymptotically to zero. Such behaviour might be represented to good approximation by the expression

$$
T_{1}(t)=T_{1} \exp \left(-\frac{t}{\tau}\right)
$$

with $\tau$ given by (8). This assumption of an exponential decay of central temperature with time is supported by the measurements of Krempl (1962), and also to some extent by those of Allan, Edels and Whittaker (1961).

Using the expression (9) for $T_{1}(t)$, the integral and constant $C$ in the numerator of equation (6) can be evaluated to give

$$
\frac{R^{2}}{\kappa \tau} \simeq \frac{T_{1} \exp (-t / \tau)-T_{0}-T_{0} \ln \left[T_{1} \exp (-t / \tau) / T_{0}\right]}{\sum_{n=1}^{\infty} a_{n}\left[T_{1} \exp (-t / \tau)-T_{0}\right]^{n}}
$$

Before going on to apply this equation to the experiment under discussion we should mention possible end effects. The ordinary theory of heat conduction (Carslaw and Jaeger 1959) suggests that if a plane, transverse to the column, is suddenly held at zero temperature-a situation corresponding roughly to that near an electrode-then, after a time $t$, the effect of this disturbance will have penetrated a distance approximately $2 \sqrt{ }\left(\kappa^{\prime} t\right)$ into the plasma, where $\kappa^{\prime}=\kappa / \rho C_{\mathrm{P}}$ is the thermal diffusivity of the plasma. Whilst this treatment is in no way exact, it suggests the extent of the region over which end effects are appreciable, and will be used to obtain numerical estimates of end effects in the following section.

\section{§5. COMPARISON WITH EXPERIMENT}

Comparison of this theory with experiment requires, first of all, explicit knowledge of the thermodynamic properties of the plasma at the temperatures involved. In the present case the electrodes are essentially inert and the plasma is assumed to be pure air at a high temperature.

Tables of the composition, density, enthalpy and specific heat of air at a pressure of one atmosphere have been calculated by Burhorn and Wienecke (1960). The enthalpy and density are shown in graphical form in figure 5 . These curves are, however, more detailed than is justified by the approximate nature of the theory. In particular, the smooth linear or parabolic temperature profile which has been assumed presupposes a similar smooth, simple variation of the thermodynamic properties of the plasma. It is thus convenient to approximate these curves by the relations

$$
\begin{aligned}
H & \simeq 5 \times 10^{3} T^{2} \\
\rho & \simeq 1.5 \times 10^{3} T^{-2} \text { for } 5000<T<10000^{\circ} \mathrm{K}
\end{aligned}
$$

or 
where c.g.s. units are used throughout. The degree of approximation of these curves to the true data is shown in figure 5 .

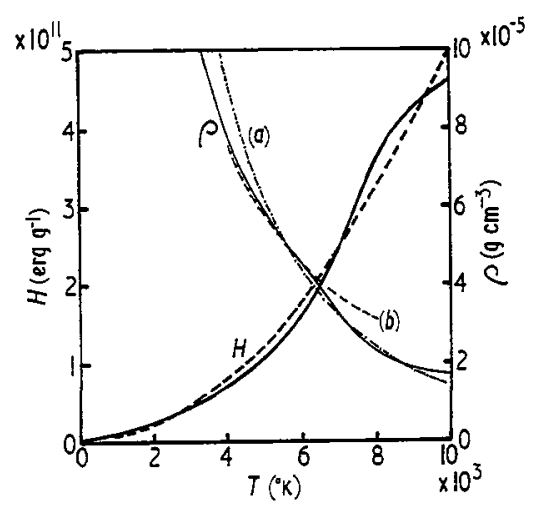

Figure 5. The enthalpy $H$ and density $\rho$ of air as functions of temperature (from tabulated values given by Burhorn and Wienecke 1960). Broken curves show the approximations

$$
\begin{aligned}
& H \simeq 5 \times 10^{3} T^{2} \mathrm{erg} \mathrm{g}^{-1} \\
& \rho \simeq 1.5 \times 10^{3} T^{-2} \mathrm{~g} \mathrm{~cm}^{-3} \\
& \rho \simeq 1.0 \times 10^{3} T^{-2}+1.5 \times 10^{-5} \mathrm{~g} \mathrm{~cm}^{-3} .
\end{aligned}
$$

The value of the thermal conductivity $\kappa$ of air at high temperatures is not easily calculated, and, because of dissociation effects, it has a large hump centred at about $7000^{\circ} \mathrm{K}$. The assumption of a smooth temperature profile implies that $\kappa$ has a simple behaviour with temperature, and requires that, in comparisons of theory with experiment, some sort of average value of $\kappa$ must be used. As will be discussed below, the temperature $T_{0}$ at the edge of the column in the experiments is probably about $4000^{\circ} \mathrm{K}$, so that it is the average value of $k$ between $4000^{\circ} \mathrm{K}$ and about $6000^{\circ} \mathrm{K}$ which must be used. Calculations by Wienecke (1956) and by Burhorn (1959) suggest that in this range $\kappa$ lies between $4 \times 10^{4}$ and $1 \times 10^{5} \mathrm{erg} \mathrm{cm}^{-1} \mathrm{sec}^{-1} \mathrm{deg}^{-1}$. For our calculations we adopt a value of $6 \times 10^{4} \mathrm{erg} \mathrm{cm}^{-1} \mathrm{sec}^{-1} \mathrm{deg}^{-1}$.

Because Armidale is about $1000 \mathrm{~m}$ above sea level, the value of $\rho$ for the conditions of the experiment should be about $10 \%$ less than that given above. In view of the uncertainty in $\kappa$, however, this small correction is not of any importance.

One further point which must be established is the value of $T_{0}$, which the experimental method recognizes as defining the boundary of the channel. The breakdown of the barium stearate layer on the anode appears to be caused by a build-up of electrons deposited on the surface of the layer by the probing pulse. Whether or not breakdown occurs is thus related to the density of electrons in the plasma, and it is reasonable that the boundary will appear at a temperature at which the concentration of electrons is a rapidly varying function of temperature. Such a natural boundary occurs near $4000^{\circ} \mathrm{K}$ in air. In this region the electrons are produced mainly by ionization of nitric oxide, and at $4000^{\circ} \mathrm{K}$ the electron density is about $10^{13} \mathrm{~cm}^{-3}$, while at $3000^{\circ} \mathrm{K}$ it has fallen to $1011 \mathrm{~cm}^{-3}$. This is a much sharper variation than occurs at any higher temperature. Rough calculations support the view that the electrons which would be drawn to the anode film by the probing pulse from a concentration of electrons of $10^{13} \mathrm{~cm}^{-3}$ in the plasma should produce a surface charge density sufficient to cause breakdown in the film. At this temperature and electron density the time for recombination of electrons 
and positive ions is probably sufficiently short to be neglected on the time scale of the thermal relaxation processes.

To extend the usefulness of the solutions, the behaviour of $R(t)$ has been plotted in figure 6 for boundary temperatures $T_{0}$ of 4000,5000 and $6000{ }^{\circ} \mathrm{K}$. Figure $6(a)$ assumes a parabolic and figure $6(b)$ a linear radial temperature profile in the arc column. In both figures the thermal conductivity $\kappa$ has been left arbitrary, and the curves are plotted in terms of the parameter $\tau$ which is fixed by the initial conditions according to equation (8). The origin of $t$ is arbitrary, and the $t / \tau$ scale can be slid along so that its origin coincides with the initial value of $T_{1}$ as shown at the top of each figure. In the region of good convergence of the denominator of (10) the curves are drawn as full lines. Broken curves are extrapolations to regions of poor convergence.
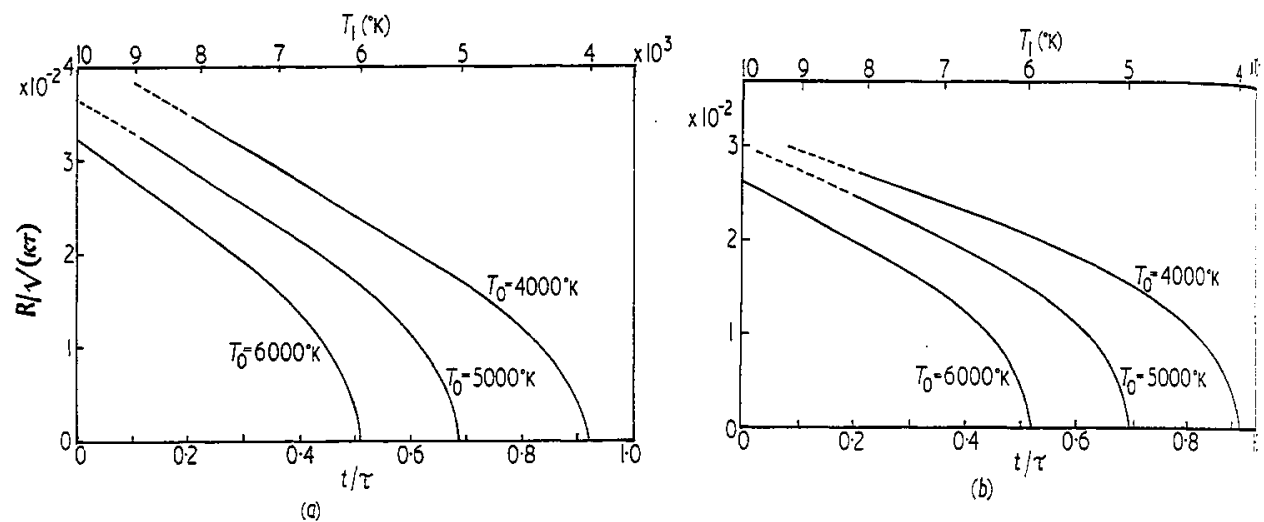

Figure 6. Decay curves calculated from equation (10) for a cylindrical plasma having a central temperature parameter $T_{1}$ and a temperature $T_{0}$ at radius $R$. $\kappa$ is the thermal conductivity at temperature $T_{0}$, and $\tau$ is a scale parameter. The origin of $t$ is arbitrary and should be chosen to correspond to the initial value of $T_{1}$ shown along the top edge of the graph. In the figures it is shown arbitrarily as corresponding to $T_{1}=10000^{\circ} \mathrm{K}$. Broken curves are extrapolations to regions of doubtful convergence.

(a) Parabolic temperature profile; (b) linear temperature profile.

It is clear that the curves in figure 6 resemble qualitatively the experimental results of figure 3 . Since the boundary temperature $T_{0}$ is quite low, a linear profile is probably a better approximation to the expected bell-shaped temperature distribution within the column than a parabola would be, and so will be assumed in what follows. One parameter is disposable, namely the central temperature $T_{1}$. Once this has been assumed then the initial radius $R(0)$ fixes $\tau$ through (8). The experimental curves suggest that thermal contraction of the column begins about $2.5 \mu \mathrm{sec}$ after the passage of the initiating pulse, and this has been chosen as the origin for $t$.

Theoretical curves using the values discussed above for $T_{0}$ and $\kappa$, and with $T_{1}$ chosen for best fit, are shown in figure 3 . The central temperatures, which will in reality be rather less than $T_{1}$, are relatively low for arcs, but it must be remembered that, in the experiment, current flows for less than $0 \cdot 1 \mu \mathrm{sec}$, and at the chosen origin $t=0$ the arc has already been extinguished for $2.4 \mu \mathrm{sec}$. At earlier times the central temperature must be very much higher than $T_{1}$.

A brief consideration of end effects on the basis of the discussion given at the end of $\S 4$ suggests that, in a time of about $12 \mu \mathrm{sec}$, detectable end effects will have penetrated about $0.03 \mathrm{~cm}$ from each electrode, and marked end effects about half this distance. 
Since changes in the radial temperature distribution are very large during this time, only large end effects are of importance. In the experiments the total column length was $0.08 \mathrm{~cm}$ so that penetration of end effects 0.01 to $0.02 \mathrm{~cm}$ from each electrode is not serious enough to invalidate the analysis.

It is also of interest to calculate the total enthalpy of the plasma column at $t=0$. Unfortunately this quantity is quite sensitive to details of the shape of the temperature profile for large values of the radius, so that only an order-of-magnitude estimate can be made. It is found that the total enthalpy of the column exceeds that of the cold gas from which it was formed by about $2 \times 10^{4} \mathrm{erg} \mathrm{cm}^{-1}$, this quantity being slightly larger for the longer initiating pulses. This result accords well with that of Somerville and Williams (1959) who found a value of $1 \times 10^{4} \mathrm{erg} \mathrm{cm}^{-1}$, for the kinetic part of this energy, by identifying the channel boundary in the early stages of expansion with the shock front resulting from the instantaneous release of this energy. The present result includes dissociation and ionization energy. The higher value associated with longer pulses may be attributed to ohmic heating of the plasma while it is carrying current.

\section{$\S 6$. CONCLUSIONS}

The measurements described above complement those previously published by Somerville and Williams to provide a fairly complete study of the evolution of the plasma channel produced by a very short spark discharge in air at atmospheric pressure. After about $2.5 \mu \mathrm{sec}$ the plasma column is essentially in pressure equilibrium with its surroundings and its subsequent decay is attributed principally to radial thermal conduction with consequent contraction of the cooling plasma.

The theory which has been developed, based upon these premises, appears to give a reasonable description of the cooling process and to yield numerical results in good accord with experiment. The theory is, almost necessarily, based upon a greatly simplified model which neglects many details of the physical situation. It seems likely that a more detailed solution would necessarily require the application of numerical techniques, with consequent loss of generality.

In the course of the discussion, explicit values have been adduced for the temperature at the centre of the column as a function of time. It would be very desirable to have a direct check on this temperature after the manner of Krempl (1962), and experimental work along these lines is now proceeding.

\section{ACKNOWLEDGMENTS}

This study is part of a programme of research supported by the Electrical Research Board of Australia.

\section{REFERENCES}

Alian, J. W. S., Edels, H., and Whittaker, D., 1961, Proc. Phys. Soc., 78, 948.

BuRHORN, F., 1959, Z. Phys., 155, 42.

Burhorn, F., and Wienecke, R., 1960, Z. Phys. Chem., 215, 269.

CARsLaw, H. S., and JAEGER, J. C., 1959, Conduction of Heat in Solids (Oxford: Clarendon Press), p. $58-60$.

DrabKINA, S. I., 1951, Zh. Eksper. Teor. Fiz., 24, 473.

KREMPL, H., 1962, Z. Phys., 167, 302.

Lavgmuir, I., 1939, Proc. Roy. Soc. A, 170, 1.

Somervilie, J. M., 1959, Proc. Phys. Soc., 74, 378.

SomprVille, J. M., and Grainger, C. T., 1956, Brit. J. Appl. Phys., 7, 109.

Somierville, J. M., and Williams, J. F., 1959, Proc. Phys. Soc., 74, 309.

Stanton, R. L., 1958, The Canadian Mineralogist, 6, 87.

WieNECKe, S., 1956, Z. Phys., 146, 39. 